\section{As possibilidades quanto à sociologia global: uma perspectiva pós-colonial*}

\author{
Gurminder K. Bhambra**
}

Resumo: Este artigo aborda a maneira como as percepções sobre a natureza globalizada do mundo em que vivemos estão começando a ter um impacto dentro da sociologia, de tal forma que esta precisa interagir não apenas com as mudanças na arquitetura conceitual da globalização, como também com o reconhecimento do valor epistemológico e da agência do mundo além do Ocidente. Examino três das principais evoluções dentro da sociologia que estão focadas nessas preocupações: primeiro, a mudança para um paradigma de modernidades múltiplas; segundo, apontamentos acerca de uma sociologia global multicultural; e, terceiro, um argumento em favor de uma abordagem global cosmopolita. Embora as três abordagens em discussão se baseiem em uma consideração do "resto do mundo", seus termos, sugiro, não estão adequados para as intenções declaradas. Nenhuma dessas respostas é suficiente no que diz respeito à sua abordagem referente a omissões anteriores e cada uma acaba retornando aos problemas da posição predominante que já vem sendo criticada por outros motivos. Em contraste, eu argumento que é somente por meio do reconhecimento da significância do "global colonial" na constituição da sociologia que é possível entender e abranger o presente necessariamente pós-colonial (e descolonial) da "sociologia global".

Palavras-chave: modernidades múltiplas; sociologia multicultural; nacionalismo metodológico; cosmopolitismo metodológico.

\section{Introdução}

E

ste artigo aborda a maneira como as percepções sobre a natureza globalizada do mundo em que vivemos estão começando a ter um impacto dentro da sociologia, de tal forma que esta precisa interagir não apenas com as mudanças no que Saskia Sassen (2007) chama de arquitetura conceitual da globalização, como também com o reconhecimento do valor epistemológico e do que Leela Gandhi (1998) define como a agência do mundo que está além do Ocidente. A ideia de uma "sociologia global," argumentarei, tem sido promovida como meio para a sociologia realizar reparos necessários por conta de uma negligência anterior para com aqueles representados como "outros" em sua construção da modernidade, apontando para um rejuvenescimento da sociologia adequada para esta nova era global. Neste artigo, examinarei três das principais evoluções dentro da sociologia que estão focadas nessas preocupações: primeiro, a mudança para um paradigma de modernidades múltiplas que se afasta de teorias anteriores da modernização linear; segundo, apontamentos acerca de uma sociologia global multicultural, levando em consideração os trabalhos de estudiosos de outras partes do mundo; e, terceiro, um argumento contra o nacionalismo metodológico percebido em relação a muitas das ciências sociais, em favor de uma abor-
Recebido: 11.11 .13

Aprovado: 15.01 .14

* Tradução de David Harrad. <davidharrad@ hotmail.com>.

** Professora de sociologia e diretora do Centro de Teoria Social da Universidade de Warwick, Reino Unido. <g.k.bhambra@ warwick.ac.uk>. 
dagem global cosmopolita. Embora as três abordagens em discussão se baseiem em uma consideração do "resto do mundo", geralmente em resposta a críticas anteriores a respeito da falta de tal interação, seus termos, sugiro, não estão adequados para as intenções declaradas. Argumentarei que nenhuma dessas respostas é suficiente no que diz respeito à sua abordagem referente a omissões anteriores e cada uma acaba retornando aos problemas da posição predominante que já vem sendo criticada por outros motivos. Em grande medida, essas abordagens replicam divisões e problemas existentes em vez de desafiá-los e resolvê-los.

Em contraponto, argumentarei que uma abordagem pós-colonial de "sociologias conectadas", com sua crítica do eurocentrismo e sua preocupação central com as histórias do colonialismo e da escravidão, proporciona recursos mais adequados para o entendimento de nosso mundo global contemporâneo. Eu argumento que é somente por meio do reconhecimento da significância do "global colonial" na constituição da sociologia que é possível entender e abranger o presente necessariamente pós-colonial (e descolonial) da "sociologia global". O reconhecimento do papel histórico do colonialismo e da escravidão na configuração do mundo moderno permite-nos examinar como esses processos histórico-mundiais têm construído nossas concepções do global em termos de hierarquias de caráter racial embutidas tanto nas instituições como no desenvolvimento de conceitos e categorias sociológicos. A reorganização do entendimento a partir da ótica da colonialidade - eu argumento reconhece a significância de um tipo específico de ordenamento hierárquico que, em grande medida, tem sido implícito na nossa disciplina e continua faltando nas três respostas em discussão. Enquanto a imaginação sociológica até agora vinha se formando em volta de determinadas transformações de hierarquias - por exemplo, da condição social (status) para a cidadania (e as questões associadas de classe e gênero nesse processo) -, a imaginação sociológica pós-colonial amplia o escopo por meio de uma análise da reprodução e transformação de hierarquias de caráter racial em escala global, argumentando que possuem significância semelhante a outras hierarquias e estão embutidas nas mesmas de forma parecida.

O surgimento e o desenvolvimento da crítica pós-colonial no âmbito das ciências sociais têm levado defensores da visão "padrão" a fazerem pequenos ajustes, embora depois sugiram que já seja algo muito conhecido. O argumento é que, embora a crítica já tenha tido força, esta dizia respeito a posições que já foram ultrapassadas. Acreditam que as pequenas modificações feitas às posições existentes sejam suficientes e de modo geral o enfoque está em mudar aplicações futuras da sociologia em consonância com essas modificações. No entanto, eu defendo que a crítica pós-colonial da sociologia ainda não foi devidamente reconhecida, muito menos ultrapassada. Ademais, qualquer transformação adequada precisaria de uma reconstrução "de frente para trás" dos nossos entendimentos históricos da modernidade e do surgimento da 
sociologia, bem como "de trás para frente" em termos de como esse entendimento recém-construído nos permitiria contemplar de forma diferente questões a respeito do presente e do futuro. Um paralelo que talvez seja útil nessa reflexão é o do feminismo e sua crítica da sociologia.

A questão nos debates feministas na sociologia não era simplesmente acerca de uma reivindicação de que a extensão empírica de problemas abordados pela sociologia precisasse ser ampliada, mas que também tópicos já existentes precisavam ser entendidos em termos da relação com questões de gênero que eram, e continuam sendo, implícitas nos mesmos. Na sua forma mais robusta, o feminismo introduziu uma reorientação conceitual da sociologia em torno da ideia do patriarcado e, na sua forma mais tênue, em torno de como a natureza das relações sociais se baseia no gênero. Essas críticas não envolviam simplesmente afirmações de que no momento do reconhecimento do gênero havíamos entrado em um mundo que a partir de então deveria ser entendido como sendo baseado em gêneros e que, no futuro, as categorias sociológicas deveriam contemplar as questões de gênero. Além disso, também se argumentava que os entendimentos estabelecidos acerca do passado eram deficientes exatamente porque o gênero era uma questão do passado (ainda que não reconhecido) e também do presente e do futuro. A necessidade da reconstrução dos objetos da sociologia não era discernível antes do impacto do feminismo sobre a sociologia e esta foi necessariamente reconstruída como consequência do encontro com a crítica feminista (Holmwood, 1995; 2001; Jackson 1999; Stanley 2000; 2005). A situação análoga em relação às críticas pós-coloniais das ciências sociais é uma estrutura social e política da modernidade que necessariamente afeta outras estruturas sociais relacionadas à modernidade e que as relações sociais são necessariamente de caráter racial ou hierarquizadas de outra maneira em termos coloniais (cf. Bhambra, 2007b). O escopo da "sociologia global", corretamente entendida, deve ser o de tratar de problemas e questões que não podem ser vistos simplesmente como consequência de manifestações da "modernidade mais recente". Uma sociologia verdadeiramente global precisaria reconhecer as histórias do colonialismo e da escravidão em qualquer tentativa de repensar a sociologia como sendo adequada para nossa era global (pós-colonial). ${ }^{1}$

\section{Modernidade, sociologia e crítica pós-colonial}

A sociologia e a modernidade, como já argumentaram muitos estudiosos, precisam ser entendidas como sendo co-constitutivas (Heilbron, 1995). ${ }^{2}$ Foi com o surgimento do que se entende ser o "mundo moderno" - os eventos conjuntos e cumulativos da Renascença e da Reforma, da Revolução Científica, da Revolução Francesa e da Revolução Industrial - que uma nova e "moderna" forma de explicação - a sociologia - surgiu para fazer sentido daquele mundo. Com efeito, fixar os parâmetros do "moderno" passou a ser definido como uma das principais tarefas da sociologia,

\footnotetext{
1. Para uma discussão do envolvimento da sociologia com questões de império e colonialismo, cf. Magubane (2005) e Go (2009).

2. Os argumentos nesta seção são desenvolvidos mais detalhadamente em Bhambra (2007a).
} 
3. Neste contexto é significativo que o questionamento de Latour (1993) quanto à ideia de modernidade - de que nunca fomos modernos - se dá a partir de uma perspectiva "antropológica", afirmando tanto a diferença quanto a falta de uma diferença fundamental entre o moderno e o que o precedeu. Contudo, na elaboração de suas redes ampliadas na construção de fenômenos sociais, o próprio Latour não vai além do Ocidente. conceitual e metodologicamente. Mesmo nos casos em que sociólogos discordaram subsequentemente sobre a natureza da modernidade, o momento em que surgiu, ou seu caráter posterior, todos concordam quanto ao seu papel central na configuração da disciplina (cf., por exemplo, Giddens 1973; Heilbron, 1995; Nisbet, 1966). Ademais, não obstante as muitas diferenças entre os sociólogos em suas tentativas de delinear a modernidade, todos concordam que a mesma é marcada por ideias de ruptura e diferença: uma ruptura temporal entre um passado pré-moderno e um presente industrial moderno, e por uma diferenciação espacial qualitativa (cultural) entre a Europa (e o Ocidente) e o resto do mundo. Visto que a sociologia foi constituída no contexto do surgimento do mundo moderno e organizada em termos de propiciar uma explicação moderna daquele mundo, não é de se surpreender que a sociologia passou a ser fortemente associada aos entendimentos sobre o que é "moderno". O "tradicional" - do qual se distinguiu o moderno - foi visto como sendo da competência da antropologia, ou de estudos da área à época (cf. Steinmetz, 2007). ${ }^{3}$ Desta forma, a própria divisão entre as disciplinas estruturou uma divisão do mundo que ocultou as interconexões que constituíam o global que estava no processo de ser dividido. De fato, reformulou essa divisão em termos de um processo de desenvolvimento que resolveria diferenças na difusão de uma modernidade que era representada como sendo histórico-mundial em sua significância.

Essa divisão - postulada como sendo explicativa e normativa - foi levada a cabo metodologicamente por meio da utilização de tipos ideais como base para a análise histórica comparativa. Os tipos ideais necessariamente abstraíam um conjunto de determinadas conexões a partir de conexões mais amplas e, ademais, sugeriam processos endógenos sui generis como parte integral das conexões abstraídas (para uma discussão mais aprofundada, cf. Holmwood e Stewart, 1991). As conexões mais frequentemente omitidas são aquelas que "conectam" a Europa e o Ocidente (o moderno) à maior parte do resto do mundo (tradição). Dessa forma, essas conexões se tornam exógenas aos processos delas abstraídas, ao mesmo tempo em que estes processos são representados como expressando um grau significativo de coerência interna, independente dessas conexões mais amplas. Assim, se estabelece na análise um enfoque predominantemente centrado na Europa, tanto metodológica quanto normativamente, e relega as contribuições não europeias a inflexões culturais específicas de estruturas preexistentes consideradas como produto da modernidade europeia (Bhambra, 2007a). A melhor exemplificação disso é a persistência da crença no milagre na Europa, quando não no milagre da Europa; isto é, segundo Weber, uma crença de que a modernidade surgiu primeiro na Europa e depois se difundiu para o resto do mundo. Embora agora seja menos provável que a associação da modernidade com a Europa possa ser apresentada como modelo normativo, mesmo assim é postulada como um fato histórico; um fato em que há uma afinidade eletiva entre as estruturas instituídas da modernidade e os valores do lluminismo atribuídos a origens europeias. 
Dessa forma, a modernidade se funde com a Europa e o processo de se tornar moderno passa a ser, pelo menos inicialmente, um processo de desenvolvimento endógeno europeu, seguido pela difusão ao resto do mundo.

A industrialização, por exemplo, é vista como um fenômeno europeu que foi difundido subsequentemente no mundo. Contudo, se tomamos as fábricas de algodão em Manchester e Lancaster como sendo emblemáticas da Revolução Industrial no Ocidente, veremos que o algodão não era uma planta nativa da Inglaterra, muito menos do Ocidente (Washbrook, 1997). Veio da Índia, assim como as tecnologias para seu tingimento e tecelagem. O algodão foi cultivado nas plantações do Caribe e da região sul dos Estados Unidos por africanos escravizados transportados até lá como parte do comércio europeu de seres humanos. A própria exportação do tecido dependia da destruição da produção local de artefatos de algodão em outras partes do mundo (Bhambra, 2007a). Assim, percebemos que a industrialização não foi apenas um fenômeno europeu ou ocidental, e sim um fenômeno que dependia de condições globais para seu próprio surgimento e articulação. A história da modernidade, contudo, como é geralmente contada, baseia-se, como argumenta Homi Bhabha (1994: 250), no "apagamento do momento colonial e pós-colonial" (cf., também, Chakrabarty, 2000). Supõe-se que o resto do mundo seja externo aos processos histórico-mundiais selecionados para consideração e, concretamente, as conexões coloniais de significância para os processos em discussão são apagadas, ou silenciadas. Eu sugiro que não se trata de um erro de conhecimento individual, e sim de algo que se torna possível pela própria estrutura disciplinar da produção do conhecimento que separa o moderno (sociologia) do tradicional e do colonial (antropologia), não deixando assim qualquer espaço para a consideração daquilo que poderia ser denominado o "moderno pós-colonial."

Ao seguir Bhabha (1994), eu argumento que o ponto de partida para qualquer entendimento da "sociologia global" há de ser uma consideração de uma história adequada para as condições sociais e políticas do presente. Estas condições não são simplesmente baseadas em entendimentos da "globalização", baseiam-se mais especificamente em um entendimento das condições globais pós-coloniais que raramente são o ponto de partida das análises sociológicas (cf. Bhambra, 2007b). Como comenta Seidman (1996: 314), por exemplo, o surgimento da sociologia coincidiu com o auge do imperialismo ocidental, no entanto "a dinâmica do império não foi incorporada nas categorias básicas, nos modelos de explicação ou nas narrativas do desenvolvimento social pelos sociólogos clássicos". Aqueles que defendem a abordagem predominante à sociologia histórica comparativa frequentemente aceitam que o eurocentrismo é um problema que às vezes tem distorcido a maneira como a modernidade tem sido conceituada dentro da sociologia. Também argumentam que o "eurocentrismo" não pode ser negado como "fato", ou que, em outras palavras, 
4. Para um aprofundamento dos argumentos apresentados nesta seção, cf. Bhambra (2007a: 56-79). as origens europeias da modernidade não podem ser negadas. Contudo, é justamente esse "fato" que é negado quando se reconhecem as interconexões globais (cf. Bhambra, 2007a; Hobson, 2004). Neste artigo argumento que persistir em ver a Europa como a "sociedade líder," para utilizar a formulação significativa de Parsons (1971), ainda que a sociedade líder dentro daquilo que agora é caracterizado como uma pluralidade globalmente constituída das "modernidades múltiplas" (por exemplo, Beck, 2000; Eisenstadt, 2000; Wittrock, 1998), mantém uma hierarquia problemática (e implicitamente normativa), baseada em uma versão historicamente inadequada do surgimento da modernidade, que não possibilita a consideração de uma sociologia propriamente global. Em uma sociedade propriamente global, as interconexões seriam reconhecidas como sendo constitutivas da modernidade e seus ordenamentos institucionais, e não vistas simplesmente como um aspecto de uma etapa subsequente da globalização.

\section{Modernidades múltiplas e variedades culturais globais}

Nos últimos anos, a teoria da modernização, com sua pressuposição da convergência global unilinear para um modelo implicitamente ocidental, tem sido superada pela abordagem baseada nas modernidades múltiplas e sua preocupação com variações culturais globais (Eisenstadt, 2000). ${ }^{4}$ Dentro dessa abordagem, entende-se que o moderno engloba caminhos divergentes, onde a variedade global de culturas leva a uma multiplicidade de modernidades. A mudança em relação à teoria inicial da modernização ocorreu, em parte, como consequência dos estudiosos começarem a perceber que as diferenças manifestas no mundo não eram, como se acreditava antes, simplesmente diferenças arcaicas que iriam desaparecer à medida que a modernização avançava. Em vez disso, há o reconhecimento de que outras sociedades poderiam modernizar-se de diferentes maneiras e que essas diferenças, para os teóricos das modernidades múltiplas, agora representam as diferentes formas de adaptação de cada sociedade específica aos processos de modernização. Ainda persiste uma crença de que a modernidade era, em suas origens, um fenômeno europeu (e ocidental), mas agora o argumento é que em sua difusão para fora ela interagiu com as diferentes tradições de várias culturas e sociedades e resultou em uma multiplicidade de modernidades não convergentes. É esta multiplicidade que é vista como separando a teoria das modernidades múltiplas da teoria anterior sobre modernização que, pode-se afirmar, era eurocêntrica em sua postulação de uma modernidade singular, para a qual se esperava que todas as demais sociedades convergissem. Este reconhecimento aparente da diferença e a inclusão estrutural da multiplicidade dentro do marco conceitual da modernidade são considerados modificações suficientes para responder à crítica pós-colonial da modernidade como sendo eurocêntrica. 
O argumento colocado por teóricos das modernidades múltiplas é que, embora a ideia de uma única modernidade, sobretudo uma que já foi alcançada na Europa, seja eurocêntrica, as teorias das modernidades múltiplas precisam, mesmo assim, tomar a Europa como o ponto de referência no seu estudo de modernidades alternativas (Eisenstadt e Schluchter, 1998: 2). Isto é uma consequência do fato de Eisenstadt e Schluchter caracterizarem a modernidade em termos de uma divisão entre sua forma institucional e um programa cultural que - sugerem - também "sofre antinomias internas e contradições, levando ao discurso crítico contínuo e a contestações políticas" (Eisenstadt, 2000: 7). Essas antinomias internas são consideradas como a base da variedade das formas da modernidade - geralmente patológicas - que subsequentemente passam a existir, como os tipos soviéticos comunistas e os tipos fascistas, nacional-socialistas (cf. Arnason, 2000). O tipo padrão da modernidade europeia é apresentado como a forma exemplar - na qual as tensões entre questões de autonomia, emancipação e reflexividade, de um lado, e questões de disciplina e controles restritivos, do outro lado, são resolvidas - e como a base da crítica de outras formas patológicas. Embora os teóricos das modernidades múltiplas apontem para o problema do eurocentrismo, ao mesmo tempo também afirmam a prioridade necessária a ser dada ao Ocidente na construção de uma sociologia histórica comparativa das modernidades múltiplas.

A sugestão por parte de teóricos das modernidades múltiplas de que a modernidade precisa ser entendida em termos de uma constelação institucional flexionada por diferenças culturais, Ihes possibilita situar a modernidade europeia - vista em termos de uma combinação única de formas institucionais e culturais - como a modernidade originária e, ao mesmo tempo, permite diferentes codificações culturais que resultam na modernidade ter se tornado múltipla. Desta forma, a Europa passa a ser a origem do tipo eurocentrado e suas pressuposições iluministas (Eisenstadt e Schluchter, 1998: 5). Ademais, argumenta-se que essas pressuposições sejam necessárias para a crítica das patologias, ao mesmo tempo em que são absolvidas de implicação na criação desses tipos patológicos. Em especial, chama a atenção que questões de colonialismo e escravidão não aparecem nem nas representações do modelo nem nas formas patológicas e, em verdade, não são consideradas parte das estruturas sociopolíticas ou econômicas da modernidade. Defender a flexão cultural das instituições permite que os teóricos das modernidades múltiplas possam apresentar a ideia de que as próprias instituições centrais não são formadas socioculturalmente. Desta forma, questões de raça e etnia, por exemplo, passam a ser vistas como limites externos das formas de mercado, ou acréscimos às mesmas, em vez de se constituírem, elas próprias, em formas de mercado. Embora uma das respostas sociológicas aos relatos convencionais sobre a modernização tenha sido argumentar que as formas institucionais centrais devam ser entendidas como sendo estruturadas por classe ou por gênero, o que continua faltando é a crítica para- 
lela de que essas formas também embutem hierarquias racializadas (cf. Bhambra, 2007b; Holmwood, 2001).

Como Arif Dirlik já argumentou, ao identificar "multiplicidade" com cultura e tradição, "a ideia de 'modernidades múltiplas' busca conter os desafios para a modernidade" - e também, eu argumentaria, os desafios para a reconfiguração substancial da sociologia - "ao conceder a possibilidade de maneiras culturalmente diferentes de ser moderno" (2003: 285), mas sem contestar o que é ser moderno e sem chamar a atenção para as interconexões sociais nas quais a modernidade foi constituída e desenvolvida. Ao manter uma matriz geral dentro da qual se localizam as particularidades - e ao identificar as particularidades com a cultura (ou com o social) e a experiência da Europa com a própria matriz geral -, os teóricos das modernidades múltiplas, de fato, têm procurado castrar qualquer desafio que pudesse ter sido levantado por uma consideração do pós-colonial. Desta forma, os teóricos das modernidades múltiplas procuram desarmar a crítica ao reconhecerem a multiplicidade e, ao mesmo tempo, manterem a estrutura fundamental do argumento original. A ideia das modernidades múltiplas pode ser argumentada de modo a representar um tipo de multiculturalismo global, no qual uma modernidade comum (eurocentrada) é flexionada por (outras) culturas diferentes. Neste contexto, é significativo que outras chamadas - aparentemente sem conexão - para uma sociologia global contenham a forma de uma chamada por uma sociologia global multicultural.

\section{Sociologia global multicultural}

Embora o argumento das modernidades múltiplas forneça uma crítica da teoria da modernização linear e se comprometa com uma reavaliação da substância das categorias sociológicas, aquilo que eu chamo de sociologia global multicultural contempla questões de epistemologia sociológica no contexto das modernidades múltiplas. Os argumentos mais recentes em prol de uma sociologia global multicultural têm surgido após a realização de duas conferências do Conselho das Associações Nacionais da Associação Internacional de Sociologia organizadas por Sujata Patel, em Miami, no ano de 2006, e por Michael Burawoy, em Taipei, em 2009. As discussões dessas conferências têm sido amplamente comentadas em revistas e outras publicações (cf., por exemplo, Burawoy et alii, 2010; Patel, 2010b), e consolidam temas de envolvimentos anteriores de sociólogos com os entendimentos e as delineações da "sociologia global". Nos anos 1980, por exemplo, houve um debate abrangente sobre as possibilidades quanto à "indigenização" das ciências sociais, centrado nos argumentos de Akinsola Akiwowo (1986; 1988). O projeto de indigenização de Akiwowo baseava-se em uma chamada pela aprendizagem a partir das tradições de várias culturas a fim de desenvolver, por meio de um processo de pesquisa e argumentação, proposições e matrizes universais que fossem adequadas para essa tarefa em uma variedade de locais. Embora as cha- 
madas pela indigenização da sociologia tenham aberto "espaços para vozes alternativas", percebeu-se que tiveram pouco impacto discernível sobre a disciplina de modo mais geral (Keim, 2011: 128; cf., também, Keim, 2008). As críticas foram descartadas por serem consideradas políticas, ou politicamente corretas, e houve pouco envolvimento com as questões epistemológicas que estavam sendo levantadas, não obstante o levantamento de questões parecidas com as críticas feministas da sociologia mais ou menos na mesma época (cf., por exemplo, Hartsock, 1984; Smith, 1987).

Os debates sobre indigenização foram seguidos em décadas subsequentes por discussões sobre o desenvolvimento de tradições autônomas ou alternativas de ciências sociais. Esses argumentos em prol de uma recém-constituída versão de sociologia global foram colocados por estudiosos como Syed Hussein Alatas (2002; 2006), Syed Farid Alatas (2006; 2010), Vineeta Sinha (2003) e Raewyn Connell (2007), e focaram a necessidade de reconhecer as origens múltiplas e globalmente diversas da sociologia. 0 debate - conforme delineado por Syed Farid Alatas (2006: 5) - teve enfoque em duas vertentes complementares: a primeira, "a falta de autonomia" das ciências sociais no Terceiro Mundo; e a segunda, "a falta de uma abordagem multicultural na sociologia". A posição comum aos diferentes argumentos levantados por esses estudiosos convergiu rumo a uma convicção quanto à importância do contexto civilizacional para o desenvolvimento de tradições autônomas, ou alternativas, das ciências sociais. Com isso, alinharam-se, fosse propositadamente ou não, com a abordagem defendida pelos teóricos das "modernidades múltiplas", onde a tradição ocidental das ciências sociais, ligada à modernidade, assume uma posição de centralidade e é considerada "o ponto de referência definitivo para o início e o progresso no desenvolvimento da sociologia" em outros lugares (Syed Farid Alatas, 2006: 10).

A autonomia das diferentes tradições baseia-se em estudos de fenômenos históricos considerados exclusivos a determinadas áreas ou sociedades. Como argumenta Syed Farid Alatas (2010: 37), as tradições autônomas precisam se "fundamentar em experiências históricas e práticas culturais locais/regionais" e também em "filosofias, epistemologias e histórias [alternativas], e nas artes". Assim, as ciências sociais ocidentais tornam-se um ponto de referência para a divergência (ou para a criatividade, conforme expressada pela apropriação das tradições ocidentais lidas por meio de contextos locais) de outras tradições autônomas, ao contrário do local de convergência (ou imitação, conforme expressada pela aplicação das tradições ocidentais a contextos locais), como se acreditava ser o caso em abordagens anteriores referentes à indigenização (que - sugeriu-se - simplesmente buscavam substituir estudiosos emigrados por estudiosos "locais" treinados nas tradições dos emigrados).

Assim como no caso das modernidades múltiplas, contudo, há pouca discussão sobre o que a aquisição dessas tradições autônomas significaria para uma sociologia global, 
além de uma mera multiplicidade. O máximo sugerido é que o desenvolvimento das tradições autônomas exigiria que uma atenção renovada fosse "dada aos sujeitos até agora fora de nosso raio de pensamento" e que isso "implicaria o reposicionamento da nossa perspectiva sociológica" (Syed Hussein Alatas, 2006: 21). Há pouca discussão, contudo, sobre o porquê desses sujeitos terem estado anteriormente fora do nosso raio de pensamento ou no que consistiria o processo de trazê-los para dentro do mesmo; as exclusões são naturalizadas e transformadas em questões de identidade, e não de metodologia ou construção da disciplina. As limitações das abordagens existentes são percebidas como resultado de sua falta de interação com estudiosos e pensadores que estão fora do Ocidente e o principal problema é entendido como a marginalização e exclusão. Assim, a solução seria uma suposta igualdade, por meio do reconhecimento da diferença, corrigindo a "ausência de pensadores não europeus" nas histórias do pensamento social e sociológico. Embora isso possa permitir a criação de uma sociologia (mais) multicultural para o futuro, faz pouco para compensar a construção problemática da disciplina da sociologia no passado (cf. Adams et alii, 2005; e para mais discussão, cf. Bhambra, 2010; 2011a).

Não é de se surpreender que a ideia de uma sociologia global multicultural, assim como as críticas feministas que a antecederam (cf. Stanley, 2000), tenha gerado alegações de um relativismo problemático percebido como debilitador da sociologia. Margaret Archer, por exemplo, eu seu discurso presidencial no Congresso Mundial da Associação Internacional de Sociologia (ISA), criticou a mudança na sociologia rumo ao que ela considerou como fragmentação e localização. Com o título "Sociologia por um só mundo: unidade e diversidade", o discurso de Archer (1991: 132) mapeou "a ironia de uma sociedade cada vez mais global que tem como resposta uma sociologia cada vez mais localizada". Piotr Sztompka, outro ex-presidente da ISA, seguiu Archer e argumentou veementemente contra a tendência de estabelecer uma sociologia global multicultural. Em uma revisão recente dos anais da conferência da ISA realizada em Taipei, Sztompka (2011) argumenta que uma ideologia em particular tem permeado a ISA - a que considera problemática a hegemonia da sociologia norte-americana e europeia; que acredita na existência das sociologias alternativas, indígenas; e vê na luta por uma sociologia global uma forma de contestar a hegemonia das formas dominantes e criar uma unidade equilibrada na disciplina. Em contraste, sua principal preocupação, seguindo Archer (1991), é destacar o fato que "somente há, e somente pode haver, uma sociologia que estuda muitos mundos sociais" (Sztompka, 2011: 389). O lugar dos sociólogos que estão fora do Ocidente, segundo ele, é suplementar as verdades do centro. Conforme ele sugere,

a contribuição mais bem-vinda dos sociólogos fora da Europa ou da América é fornecer evidências, intuições heurísticas, modelos e hipóteses bem estudados e inspirados localmente para acrescentar ao banco de conhecimentos sociológicos que é universal (2011: 393). 
Há pouco entendimento de que os novos conhecimentos assim gerados possam de alguma forma reivindicar a reconstrução dos conceitos e das categorias sociológicos e portanto manter uma única sociologia; isto é, uma sociologia reconstruída com base nesses novos insights. É assim, não obstante a aceitação na versão ortodoxa de uma explicação das origens da sociologia em um momento de "descentralização" da Europa por parte de sociedades localizadas em suas fronteiras do noroeste. Uma descentralização das epistemologias sociológicas é considerada uma questão pontual, o que é irônico, uma vez que as condições sociológicas das preocupações atuais sobre a globalização se parecem muito com uma mudança geopolítica semelhante no poder para aquele que acompanhou o surgimento da modernidade conforme apresentado na literatura padrão.

\section{Cosmopolitanismo global}

Enquanto Archer (1991) e Sztompka (2011) têm criticado a mudança rumo a uma sociologia global multicultural a partir de uma perspectiva a considerar adequadas as formas existentes dos entendimentos sociológicos, outros têm feito críticas delineando uma posição alternativa. Talvez a articulação mais persuasiva em prol de uma alternativa para a sociologia global multicultural se encontre nas reivindicações por um novo universalismo de uma sociologia globalmente cosmopolita conforme proposta por Ulrich Beck (2000; 2006). Seu argumento reconhece até certo ponto o "localismo" do centro, mas o faz enquadrando-o como uma restrição para acontecimentos futuros (vindos de outro lugar) como veremos na seção a seguir. Para Beck, o problema é como evitar o relativismo dos conhecimentos locais, incluindo o da sociologia ocidental, e não como aprender a partir de conhecimentos locais existentes em outros lugares.

No decorrer da primeira década do século XXI, Beck (2000; 2006) tem apresentado uma abordagem cosmopolita para interagir criticamente com a globalização e ir além das limitações das abordagens disciplinares estadocêntricas típicas das ciências sociais e políticas. ${ }^{5}$ Beck sugere que a sociologia delimita o objeto de seu estudo dentro de fronteiras nacionais, demonstrando um nacionalismo metodológico ultrapassado, e não dentro do contexto mais apropriado da "sociedade mundial". Como consequência, a sociologia é menos capaz de interagir com o "número cada vez maior de processos sociais que são indiferentes em relação às fronteiras nacionais" (Beck, 2000: 80). Essa era global, para esse autor, é marcada por uma transição da "primeira era da modernidade", que foi estruturada por Estados-nação, para uma "segunda era" cosmopolita, na qual "rompe-se a pretensão Ocidental de monopolizar a modernidade e se vislumbram a história e a situação de modernidades divergentes em todas as partes do mundo" (Beck, 2000: 87). Assim, a era global é percebida necessariamente como era multicultural, dado que se afirma que as modernidades múltiplas 
são a expressão das diferenças culturais. Com isso, Beck segue a abordagem dos teóricos das modernidades múltiplas na análise geral destes, mas sua chamada por uma segunda era da modernidade, e o que se segue disso - uma chamada por uma sociologia cosmopolita - é distintiva.

Beck $(2000,2006)$ não só argumenta que agora a modernidade é múltipla, como também sugere que os conceitos que vinham sendo utilizados no desenvolvimento dos entendimentos sociológicos na primeira era agora não são mais adequados para a tarefa do entendimento da modernidade em sua segunda era. Isto é sobretudo uma consequência do fato de que os conceitos-padrão das ciências sociais foram desenvolvidos para entender um mundo composto por Estados-nação. Agora que estamos na segunda era - a era global - da modernidade, Beck argumenta que tais conceitos não são mais apropriados. Em seu lugar, faz-se necessário um novo conjunto de categorias e conceitos que surgiriam a partir da reflexão sobre esta nova era cosmopolita da modernidade, representada por mudanças rumo a uma sociedade mundial. Embora eu também tenha argumentado que os conceitos sociológicos são inapropriadamente restritos - especificamente, que são "metodologicamente eurocêntricos", em vez de metodologicamente nacionalistas - isto não é algo que apenas agora está se tornando um contratempo, à medida que uma suposta "primeira modernidade" cede o lugar para um mundo contemporâneo agora globalizado. No mínimo, poder-se-ia argumentar que a "primeira modernidade" foi caracterizada tanto por impérios e blocos regionais quanto por Estados-nação (cf. também Wimmer e Schiller, 2003). Como consequência, defendo que os conceitos da "primeira era" eram tão inadequados em sua própria época quanto se alega que são hoje e que precisam de uma reconstrução mais abrangente que essa sugerida por Beck.

Beck (2002) vê o cosmopolitanismo - e a reconstrução da sociologia por meio de um paradigma cosmopolita - como uma questão do presente e do futuro. Não há discussão em sua obra sobre uma reflexão quanto ao cosmopolitanismo na história e sobre uma nova análise do passado da sociologia à luz dessa perspectiva. Ademais, há pouco reconhecimento de que se determinados entendimentos são vistos como problemáticos hoje, também é provável que tenham sido problemáticos no passado e, portanto, precisam de uma revisão mais abrangente do que a proposta pelo autor. Com efeito, Beck argumenta que não está interessado na memória do passado global, mas simplesmente em como uma visão de um futuro cosmopolita poderia ter um impacto na política do presente. Parece que acredita que seja possível discutir "as implicações atuais de um futuro globalmente moldado" (Beck, 2002: 27) sem levar em consideração como os legados do passado moldaram o presente. Ele simplesmente descarta as desigualdades historicamente herdadas oriundas dos legados do colonialismo, do imperialismo e da escravidão trazidos pela Europa e segue em frente para imaginar um mundo separado da resolução dessas desigualdades. Em 
contraste, eu argumentaria que qualquer teoria que busque responder à questão de "como vivemos no mundo" não pode tratar como irrelevante a configuração histórica daquele mundo (para uma discussão, cf. Trouillot, 1995). Assim, argumento que a abordagem cosmopolita de Beck é tão limitada quanto as abordagens estadocêntricas que ele critica, justamente na maneira como relega ao passado a pertinência destes conceitos, objetando que o que está em questão é simplesmente a aplicação dos mesmos ao presente e ao futuro (para uma discussão adicional, cf. Bhambra, 2011b; e Patel, 2010a).

Por último, os argumentos de Beck em prol de uma sociedade cosmopolita continuam a tomar perspectivas ocidentais como foco dos processos globais, e a Europa como origem de uma modernidade subsequentemente globalizada. Sua versão particular do cosmopolitanismo - eu sugeriria - é uma expressão do eurocentrismo cultural disfarçado de inclusão global em potencial; digo em potencial porque tal inclusão depende dos "outros" serem incluídos nos "nós" conforme definidos por Beck (2002). Não é uma inclusão que reconhece que os "outros" estavam presentes, ainda que marginalizados e silenciados, dentro das matrizes-padrão do entendimento; tampouco é uma inclusão que procura estabelecer o cosmopolitanismo de baixo para cima (para entendimentos propriamente cosmopolitas do cosmopolitanismo (cf. Lamont e Aksartova, 2002; Mignolo, 2000; Pollock et alii, 2000), ao contrário, para Beck, uma sociologia cosmopolita é uma injunção normativa que determina como os outros devem ser incluídos e como os outros devem conviver conosco nesta nova era globalizante. Sua hostilidade para com os outros não encontra melhor exemplificação do que no título de seu artigo: "Sociedade cosmopolita e seus inimigos." Em contraposição, sugiro que uma sociologia global aberta a diferentes vozes provincializaria os entendimentos europeus em sua abordagem do global e criaria um novo universalismo baseado em uma sociologia reconstruída da modernidade.

\section{Rumo a uma sociologia global pós-colonial}

Todas as diferentes abordagens discutidas acima - modernidades múltiplas, sociologia multicultural, cosmopolitanismo - tentam lidar com duas questões principais em sua afirmação quanto a uma sociologia global. Primeiro, como a sociologia pode responder às críticas feitas por teóricos pós-coloniais, de que não contempla questões relativas à diferença conforme ela se manifesta no mundo; e, segundo, como a sociologia pode tornar-se relevante para um mundo objeto de um novo entendimento em termos globais. A principal maneira de se lidar com a primeira questão é por meio de uma abordagem que agrega e celebra uma pluralidade contemporânea de culturas e vozes. O paradigma das modernidades múltiplas, por exemplo, reconhece a diversidade de culturas globalmente localizadas e aceita a possibilidade de formas culturalmente diversas de ser moderno. Esses aspectos, da multiplicidade (em vez 
de singularidade) e da divergência (em vez de convergência), são julgadas suficientes para responder a críticas anteriores. No entanto, há pouco reconhecimento da presença desses "outros" na história da modernidade conforme entendida em sua forma originária. Os eventos histórico-mundiais reconhecidos na constituição da modernidade permanecem centrados em uma história europeia estreitamente definida e não há lugar para as histórias mais amplas do colonialismo ou da escravidão em seus entendimentos do surgimento do moderno. Essa deficiência das modernidades múltiplas se replica na mudança para as sociologias globais múltiplas, ou multiculturais, onde a centralidade do Ocidente permanece mantida e onde se permite que novas vozes suplementem as verdades já existentes acerca de uma modernidade eurocentrada, mas não se permite que as reconstruam. Se o novo cosmopolitanismo na "era da segunda modernidade" aparece diferente, é somente porque se evita o multiculturalismo, enquanto se aceita paradoxalmente as premissas conceituais e metodológicas do paradigma das modernidades múltiplas. Como observa Holmwood (2007: 55), embora os estudiosos permitam novas vozes (pós-coloniais) dentro da sociologia, seus entendimentos do esforço sociológico são tais que essas novas vozes "não afetam suas construções anteriores". Toda reconstrução deve ser aplicada ao futuro ao mesmo tempo em que mantém a pertinência das interpretações e dos entendimentos conceituais do passado.

No seu lidar com o global, as três abordagens o consideram constituído por meio de conexões contemporâneas entre o que são apresentados como contextos civilizacionais historicamente separados. Nenhuma das abordagens leva em consideração as histórias do colonialismo e da escravidão como elementos centrais do desenvolvimento "global" e, portanto, trabalham com um entendimento empobrecido que vê o global apenas como fenômeno de relevância recente. O cosmopolitanismo global de Beck, por exemplo, reconhece que os conceitos sociológicos são inadequados para a era atual, mas não reconhece o "global" como sendo constituído historicamente. Ao contrário, Beck simplesmente preocupa-se com o surgimento de uma nova era global cosmopolita e uma sociologia cosmopolita adequada para novos desafios no futuro. De modo parecido, as chamadas por uma sociologia global multicultural, na qual as vozes da periferia entrariam em debates com o centro, baseiam-se na ideia de que a sociologia poderia ser diferente no futuro, mas com pouco reconhecimento de que para isso acontecer a sociologia também precisaria relacionar-se diferentemente com seu próprio passado. Diversamente, eu argumento que para poder responder ao que é considerado problemático dentro dos entendimentos contemporâneos da sociologia, precisamos começar com uma análise da maneira como a sociologia entende o passado e como isso influencia sua configuração de categorias e conceitos no presente. Eu proponho que a principal questão é o fato de não tratar da omissão do global colonial com base em entendimentos sobre como o global veio a ser constituído como tal. 
Ao silenciar o passado colonial no âmbito da narrativa histórica central à formação da sociologia, também se desconsidera o presente pós-colonial da Europa (e do Ocidente). Como consequência, as tentativas sociológicas de tratar do "recém"-global são interpretadas erroneamente e assim são inadequadas para uma abordagem correta dos problemas em comum entre nós. Ao aceitar como adequadas versões sociológicas que excluem considerações do mundo baseadas em entendimentos de processos histórico-mundiais, perpetua-se uma forma de etnocentrismo. No entanto, como argumenta Bhabha (1994: 244), mudar a perspectiva por meio da qual visualizamos os eventos da modernidade obriga-nos a considerar a questão da agência subalterna e a perguntar: "o que é esse 'agora' da modernidade? Quem define qual é o presente a partir do qual falamos?" Essa provocação convida-nos a reanalisar o paradigma conceitual da modernidade a partir das perspectivas daqueles "outros" geralmente relegados à margem, quando não de todo excluídos. A tarefa - conforme Bhabha (1994: 7) - é assumir a responsabilidade pelos passados não mencionados e não representados existentes no nosso presente global e reconstruir os entendimentos do presente para que sejam adequados para aquele passado; e - eu acrescentaria - reconstruir os entendimentos do passado para que sejam adequados para o presente que compartilhamos.

Um exemplo disso seria o dos Estados-nação do Ocidente confrontarem suas histórias coloniais e imperiais (e assim reconhecerem seu presente pós-colonial), identificando o "influxo de migrantes e refugiados pós-guerra" como parte de "uma narrativa indígena ou nativa inerente à identidade nacional" (Bhabha, 1994: 6, grifos meus; cf., também, Amin, 2004). Da mesma forma que na literatura sociológica padrão, a industrialização é representada como endógena e sua ampliação como difusão, de modo geral a migração também tem sido vista como processo ao mesmo tempo exógeno e também subsequente à formação de Estados-nação. A ideia da comunidade política enquanto ordem política nacional tem sido central ao autoentendimento europeu, e permanece presente nas três abordagens sociológicas discutidas neste artigo. No entanto, a maioria dos Estados europeus, além de terem sido Estados coloniais e imperiais, também eram Estados nacionais - muitas vezes antes ou concomitantemente ao processo de se tornarem Estados nacionais - e por isso a comunidade política do Estado era muito mais ampla e mais (e diferentemente) estratificada do que geralmente se reconhece agora. Ao situar a migração como sendo subsequente à formação de Estados-nação, os próprios migrantes são situados como recém-chegados e sua participação naquela comunidade é considerada como diferente em relação àqueles aceitos como naturais (cf. Wimmer e Schiller, 2003). Dessa forma, na medida em que os migrantes muitas vezes são marcados racialmente, os entendimentos sobre raça e etnia passam a ser associados a questões relativas à sua distribuição posterior dentro de uma comunidade política - como "minorias" - em vez de se basearem em uma análise de seu papel constitutivo na formação dessas 
comunidades. Argumenta-se que o "caráter" essencial dessas comunidades se forma independentemente dos processos pelos quais os migrantes passam a ser ligados aos novos locais em que fixaram residência. Uma abordagem mais apropriada seria situar os migrantes dentro dos sistemas mais amplos da formação de Estados-nação no contexto de Estados imperiais e regimes coloniais para que sejam assim entendidos como integrando tais processos em vez de serem considerados com acréscimos subsequentes aos mesmos.

A mudança rumo ao global, conforme exemplificada pelas abordagens aqui expostas, é apresentada como fenômeno novo dentro da sociologia. Contudo, como procurei demonstrar, essas abordagens apenas perpetuam matrizes analíticas anteriores associadas a entendimentos sobre o moderno eurocentrado. Ao substituir o "moderno" pelo "global", naturaliza-se uma história sociológica cada vez mais contestada, o que permite que os sociólogos evitem a questão fundamental da relação entre modernidade e sociologia. Dessa forma, as histórias globais das interconexões coloniais entre o que são apresentadas como modernidades separadas continuam a ser deletadas das considerações históricas e analíticas. Como consequência, percebe-se que os entendimentos sobre a "sociologia global" surgem por meio do acréscimo de "novos" conhecimentos oriundos de lugares diferentes com pouca consideração quanto às interconexões que já existem há muito tempo entre os locais em que os conhecimentos são construídos e produzidos. Tampouco há reconhecimento de que a sociologia global precisaria que a própria sociologia fosse repensada de frente para trás, em termos de como suas principais categorias têm sido constituídas no contexto de determinadas narrativas históricas, e também de trás para frente em termos das implicações adicionais de sua reconstrução. Em contraste, uma abordagem pós-colonial à sociologia histórica requer a contemplação das histórias do colonialismo e dos impérios na configuração dos entendimentos do global. A perspectiva não é de abraçar o relativismo, mas de um reconhecimento de que uma sociologia verdadeiramente global com a intenção de ser universal derivar-se-á da reconstrução dos entendimentos atuais à luz de novos conhecimentos do passado e do presente.

\section{Agradecimentos}

Meus agradecimentos a John Holmwood, Ipek Demir e Vicky Margree por seus comentários e sugestões em relação a este artigo. Eventuais erros que tenham permanecido são meus. 
Abstract: This article addresses the way in which perceptions about the globalized nature of the world in which we live are beginning to have an impact with in sociology such that sociology has to engage not just with the changing conceptual architecture of globalization, but also with recognition of the epistemological value and agency of the world beyond the West. I address three main developments within sociology that focus on these concerns: first, the shift to a multiple modernities paradigm; second, a call for a multicultural global sociology; and third, an argument in favor of a global cosmopolitan approach. While the three approaches under discussion are based on a consideration of the "rest of the world", their terms, I suggest, are not adequate to the avowed intentions. None of these responses is sufficient in their address of earlier omissions and each falls back into the problems of the mainstream position that is otherwise being criticized. In contrast, I argue that it is only by acknowledging the significance of the "colonial global" in the constitution of sociology that it is possible to understand and address the necessarily postcolonial (and decolonial) present of "global sociology".

Keywords: multiple modernities; multicultural sociology; methodological nationalism; methodological cosmopolitanism.

\section{Referências}

AdAms, J.; Clemens, E. S.; Orloff, A. S. (Eds.). Remaking modernity: politics, history and sociology. Durham (NC): Duke University Press, 2005.

Akıwowo, A. A. Contributions to the sociology of knowledge from an African oral poetry. International Sociology, 1 (4). Sydney, 1986, p. 343-358.

- Universalism and indigenization in sociological theory: introduction. International Sociology, 3 (2). Sydney, 1988, p. 155-160.

AlATAS, S. F. The development of an autonomous social science tradition in Asia: problems and prospects. Asian Journal of Social Science, 30 (1). Cingapura, 2002, p. 150-157.

- Editorial introduction: The idea of autonomous sociology: Reflections on the state of the discipline. Current Sociology, 54 (1). London: Sage Publications, 2006, p. 5-6.

Religion and reform: two exemplars for autonomous sociology in the nonwestern context. In Patel, S. (Ed.). The ISA handbook of diverse sociological traditions. London: Sage Publications, 2010.

ALATAS, S. H. The autonomous, the universal and the future of sociology. Current Sociology, 54 (1). London: Sage Publications, 2006, 7-23.

Amin, A. Multi-ethnicity and the idea of Europe. Theory, Culture and Society, 21 (2). London: Sage Publications, 2004, p. 1-24.

ARCHER, M. S. Presidential address: sociology for one world - Unity and diversity. International Sociology, 6 (2). Sydney, 1991, p. 131-147. 
ARnASON, J. Communism and modernity. Daedalus: Multiple Modernities, 129 (1). Cambridge (MA), 2000, p. 61-90.

BECK, U. The cosmopolitan perspective: sociology of the second age of modernity. British Journal of Sociology, 51 (1). London, 2000, p. 79-105.

- The cosmopolitan society and its enemies. Theory Culture Society, 19 (1-2). London: Sage Publications, 2002, p. 17-44.

Cosmopolitan vision. Cambridge: Polity Press, 2006.

BHABHA, H. K. The location of culture. London: Routledge, 1994.

BHAMBRA, G. K. Rethinking modernity: postcolonialism and the sociological imagination. Basingstoke: Palgrave MacMillan, 2007a.

Sociology and postcolonialism: another "missing" revolution? Sociology, 41 (5). London: Sage Publications, 2007b, p. 871-874.

- Historical sociology, international relations and connected histories. Cambridge Review of International Affairs, 23 (1). Cambridge, 2010, p. 127-143.

. Historical sociology, modernity, and postcolonial critique. American Historical Review, 116 (3). Oxford, 2011a, p. 653-662.

- Cosmopolitanism and postcolonial critique. IN Rovisco, M.; NoWICKA, M. (Eds.). Companion to cosmopolitanism. Farnham: Ashgate, 2011b, p. 313-328.

Burawoy, M.; Chang, M.-K.; HSIEH Michelle, F.-Y. (Eds.). Facing an unequal world: challenges for a global sociology. v. 1-3. Taiwan, Jointly published by the Institute of Sociology at Academia Sinica, Council of National Associations of the International Sociological Association, and Academia Sinica, 2010.

Chakrabarty, D. Provincializing Europe: postcolonial thought and historical difference. Princeton (NJ): Princeton University Press, 2000.

CONNELL, R. Southern theory: the global dynamics of knowledge in social science. Cambridge: Polity Press, 2007.

DIRLIK, A. Global modernity? Modernity in an age of global capitalism. European Journal of Social Theory, 6 (3). London: Sage Publications, 2003, p. 275-292.

EISENSTADT, S. N. Multiple modernities. Daedalus: Multiple Modernities, 129 (1). Cambridge (MA), 2000, p. 1-29.

EISENSTADT, S. N.; SCHLUCHTER, W. Introduction: paths to early modernities - A comparative view. Daedalus: Early Modernities, 127 (3). Cambridge (MA), 1998, p. 1-18. 
GANDHI, L. Postcolonial theory: a critical introduction. Edinburgh: Edinburgh University Press, 1998.

GIDDENS, A. Capitalism and modern social theory: an analysis of the writings of Marx, Durkheim and Max Weber. Cambridge: Cambridge University Press, 1973.

Go, J. The "new" sociology of empire and colonialism. Sociology Compass, 3. Hoboken (NJ), 2009, p. 1-14.

HARTSOCK, N. C. M. The feminist standpoint: developing the ground for a specifically feminist historical materialism. Money, sex, and power: toward a feminist historical materialism. Boston, MA: Northeastern University Press, 1984.

HeILBRon, J. The rise of social theory. Cambridge: Polity Press, 1995.

HOBSON, J. The Eastern origins of the West. Cambridge: Cambridge University Press, 2004.

HOLMWOOD, J. Feminism and epistemology: what kind of successor science? Sociology, 29 (3). London: Sage Publications, 1995, p. 411-428.

- Gender and critical realism: A critique of Sayer. Sociology, 35 (4). London: Sage Publications, 2001, p. 947-965.

- Sociology as public discourse and professional practice: A critique of Michael Burawoy. Sociological Theory, 25 (1). London: Sage Publications, 2007, p. 46-66.

HOLMWOOd, J.; STEWART, A. Explanation and social theory. London: Macmillan, 1991.

JACKSON, S. Feminist sociology and sociological feminism: recovering the social in feminist thought. Sociological Research Online, 4(3). 1999. Retrieved from <http://www. socresonline.org.uk/4/3/jackson.html>.

KEIM, W. Social sciences internationally: the problem of marginalization and its consequences for the discipline of sociology. African Sociological Review, 12 (2). Dacar, 2008, p. 22-48.

. Counterhegemonic currents and Internationalization of sociology: theoretical reflections and an empirical example. International Sociology, 26 (1). Sydney, 2011, p. 123-145.

LAMONT, M.; AKSARTOVA, S. Ordinary cosmopolitanisms: strategies for bridging racial boundaries among working-class men. Theory, Culture and Society, 19 (4). London: Sage Publications, 2002, p. 1-25.

LATOUR, B. We have never been modern. Hertfordshire: Harvester Wheatsheaf, 1993. 
MAGUBANE, Z. Overlapping territories and intertwined histories: Historical sociology's global imagination. In AdAMS, J.; CLEMENS, E.; ORLOFF, A. (Eds.). Remaking modernity: politics, history, sociology. Durham (NC): Duke University Press, 2005, p. 92-108.

MIGNOLO, W. D. The many faces of cosmo-polis: border thinking and critical cosmopolitanism. Public Culture, 12 (3). Durham (NC), 2000, p. 721-728.

NISBET, R. A. The sociological tradition. New York: Basic Books Inc, 1966.

Parsons, T. The system of modern societies. Englewood Cliffs (NJ): Prentice-Hall Inc, 1971.

PATEL, S. The imperative and the challenge of diversity: reconstructing sociological traditions in an unequal world. In BURAWOY, M.; CHANG, M.-K.; FEI-YU HSIEH, M. (Eds.). Facing an unequal world: challenge for a global sociology. Taipei: Academica Sinica and International Sociological Association, 2010a, pp. 48-60.

- (Ed.). The ISA handbook of diverse sociological traditions. London: Sage Publications, 2010b.

Pollock, S.; Bhabha, H. K.; Breckenbridge, C. A.; Chakrabarty, D. Cosmopolitanisms. Public Culture, 12 (3). Durham (NC), 2000, p. 577-579.

SASSEN, S. A sociology of globalization. "Contemporary Society Series". New York: W.W. Norton and Co., 2007.

SEIDMAN, S. Empire and knowledge: more troubles, new opportunities for sociology. Contemporary Sociology, 25 (3). London: Sage Publications, 1996, p. 313-316.

SINHA, V. Decentring social sciences in practice through individual acts and choices. Current Sociology, 51 (1). London: Sage Publications, 2003, p. 7-26.

SMITH, D. The everyday world as problematic: a feminist sociology. Buckingham: Open University Press, 1987.

StANLEY, L. For sociology, Gouldner's and ours. In ELDRIDGE, J.; MACINNES, J.; SCOTT, S.; WARHURST, C.; WITZ, A. (Eds.). For sociology: legacies and prospects. Durham (NC): Sociology Press, 2000.

A child of its time: hybridic perspectives on othering in sociology. Sociological Research Online, 103, 2005. Retrieved from <http://www.socresonline.org.uk/10/3/ stanley.html>.

SteinmetZ, G. Transdisciplinarity as a nonimperial encounter. Thesis Eleven, 91 (1). Sage Publications, 2007, p. 48-65. 
SzTOMPKA, P. Another sociological utopia. Contemporary Sociology, 40 (4). London: Sage Publications, 2011, p. 388-396.

TROUILLOT, M.-R. Silencing the past: power and the production of history. Boston (MA): Beacon Press, 1995.

WASHBROOK, D. A. From comparative sociology to Global History: Britain and India in the pre-history of modernity. Journal of Economic and Social History of the Orient, 40 (4). Amsterdam, 1997, p. 410-413.

Wimmer, A.; SCHILleR, N. G. Methodological nationalism, the social sciences, and the study of migration: An essay in historical epistemology. International Migration Review, 37 (3). New York, 2003, p. 576-610.

WITTROCK, B. Early modernities: varieties and transitions. Daedalus: Early Modernities, 127 (3). Cambridge (MA), 1998, p. 19-40. 